\title{
Escalas de desenvolvimento infantil e o uso com bebês
}

\section{Employment of scales to evaluate babies' development}

\author{
Olga Maria Piazentin Rolim Rodrigues ${ }^{1}$
}

\begin{abstract}
RESUMO
O presente estudo ${ }^{2}$ pretendeu descrever as escalas (testes ou inventários) comumente utilizadas para a avaliação do desenvolvimento de bebês, em termos de objetivos, população a quem se destina, material sugerido, o que avaliam e critérios de avaliação. Pretendeu, também, analisar estudos que utilizaram os instrumentos aqui arrolados, a partir do final da década de 90, considerando os objetivos, a população avaliada e os principais resultados obtidos e, finalmente, identificar profissionais da saúde que têm feito uso deles, a partir dos artigos publicados. O estudo aponta para o papel importante da avaliação de bebês como fonte de informação que subsidia tomadas de decisão acerca da elaboração de programas de intervenção precoce ou essencial. Todavia, observa-se a escassez de instrumentos padronizados para a população infantil brasileira.
\end{abstract}

Palavras-chave: escalas de desenvolvimento infantil; desenvolvimento de bebês; estimulação essencial; intervenção precoce.

\begin{abstract}
The present study aimed to describe the scales (tests or inventories) commonly used to evaluate the development of babies, in terms of objectives, population in which it is destined to, suggested material, what is evaluated and evaluation criteria. It also intended to analyze studies which employed instruments here described, from the late $90 \mathrm{~s}$, considering the objectives,
\end{abstract}

${ }^{1}$ Doutora em Psicologia Experimental, pela Universidade de São Paulo. Professora Livre-Docente da Universidade Estadual Paulista "Julio Mesquita Filho" (UNESP), Brasil. E-mail: olgarolim@fc.unesp.br.

${ }^{2} \mathrm{O}$ presente artigo é parte da tese de Livre-Docência da autora. 
the population evaluated, and the main results obtained and, finally, it aimed to identify health professionals who have employed it, since the articles published. The study highlights the important role of evaluating babies, as information source which help decision taking about the elaboration of essential or early intervention programs. However, it is difficult to find standardized instruments for Brazilian child population.

Keywords: scales of infant development; babies'development; essential stimulation; early intervention.

Os instrumentos como testes, escalas ou inventários para avaliar o desenvolvimento de bebês têm sido utilizados em pesquisas aplicadas, clínicas e estabelecimentos educacionais, subsidiando a implementação de programas de estimulação precoce, orientando o planejamento de ações pontuais com crianças e seus cuidadores (NUNES; SISDELLI; FERNANDES, 1995). No entanto, para cumprir esse papel, são necessários instrumentos fidedignos na tarefa de identificar as defasagens comportamentais apresentadas. Os resultados obtidos podem, então, indicar aspectos, no repertório infantil, passíveis de intervenção direta e, também, dar indícios de possíveis causas que podem ser atribuídas a fatores maternos (interação pobre ou inadequada, falta de informações, crenças equivocadas, presença de fatores emocionais como ansiedade, depressão, etc.) ou, ainda, ao contexto social, como falta de creches, serviços de saúde insuficientes, entre outros.

Linhares, Carvalho, Bordin e Jorge (1999) defendem a utilização de escalas como uma medida sistemática de avaliação que permite comparar o desenvolvimento do bebê à norma padrão. Todavia, alertam para a importância de outras informações que devem ser consideradas, como a rotina e as interações familiares com o bebê, entre outras.

Alguns instrumentos têm sido utilizados em estudos do desenvolvimento infantil, como as Escalas Bayley de Desenvolvimento Infantil, a Escala do Desenvolvimento do Bebê no primeiro ano de vida, o Teste Denver, a Escala de Avaliação do Desenvolvimento Psicomotor Infantil (EADP), a Alberta Infant Motor Scale (AIMS), a Movement Assessment Infant (MAI) e o Inventário Portage Operacionalizado.

Ainda que ciente dos estudos realizados internacionalmente com estes instrumentos, optou-se, neste artigo, por priorizar estudos nacionais e realizados com bebês ou com crianças mais velhas, desde que analisando os efeitos de condições de risco presentes no primeiro ano de vida, característicos da nossa população.

Campos, Gonçalves e Santos (2004) diferenciam os instrumentos de avaliação de desenvolvimento quanto à sua utilização. Podem ser usados como 
triagem, na identificação de condição de risco para o desenvolvimento e para subsidiar a implantação de programas de estimulação precoce. Pesquisadores têm lançado mão do uso de escalas para avaliar a pertinência e a adequabilidade de programas de intervenção implementados em medidas pré e pós-teste, ou utilizando grupos controle, assim como para avaliar o peso que inúmeras variáveis podem ter sobre o desenvolvimento de crianças (ALVES et al., 1997; CHIARATTI; SPROCATTI; PIOVESANA, 2001; LINHARES et al., 2000; MARTINS; LINHARES; MARTINEZ, 2005, entre outros).

Quanto à natureza dos itens avaliados, podem ser gerais, de áreas específicas ou, ainda, compostos por conjuntos de áreas específicas. O Teste de Denver, as Escalas Bayley, a Escala de Avaliação do Desenvolvimento Psicomotor Infantil (EADP) e o Inventário Portage Operacionalizado são instrumentos de avaliação geral do desenvolvimento. Alguns instrumentos avaliam áreas específicas do desenvolvimento, como a Alberta Infant Motor Scale (AIMS) e a Movement Assessment Infant (MAI), bastante utilizados para a identificação de atraso motor em crianças em condição de risco. Possibilitam, também, a implementação de programas a partir dos seus resultados.

As Escalas Bayley de Desenvolvimento Infantil (Bayley Scales of Infant Development) foram descritas, inicialmente em 1933, com o objetivo de realizar o diagnóstico evolutivo do desenvolvimento (BAYLEY, 1933, citada por CAMPOS; GONÇALVES; SANTOS, 2004). Foram revisadas em 1969, em 1993 (Escalas Bailey II) e em 2006 (Escalas Bayley III), nos Estados Unidos (SILVA et al., 2011). Elas constituem-se em instrumento adequado para a avaliação de crianças de um a 42 meses de idade (com e sem deficiências) e são amplamente reconhecidas e utilizadas em estudos sobre o desenvolvimento infantil. Fornecem resultados confiáveis e válidos e sua utilidade como instrumento de pesquisa tem recebido grande suporte da comunidade científica. Avaliam cinco domínios do desenvolvimento: cognitivo, motor, linguístico, socioemocional e comportamento adaptativo. São de fácil aplicação, com duração média de 30 minutos, envolvendo atividades e brinquedos que facilitam a interação bebê-examinador. Fornecem informações sobre o desenvolvimento extremamente importantes para orientar o trabalho de intervenção e estimulação precoce. Mais recentemente observa-se a existência de escalas que possibilitam três conjuntos de informações: a Escala Mental (IDM), com 163 itens; a Escala Motora (IDP), com 81 itens, e a Escala Comportamental Infantil (RCI), com 30 itens. A Escala Mental avalia a acuidade perceptiva visual, discriminação entre objetos, habilidades de resolução de problemas, linguagem e memória. A Escala Motora avalia o controle postural e a motricidade apendicular. A Escala Comportamental avalia o seguimento de instruções, atitudes e energia durante o teste, entre outros comportamentos sociais. Em geral, os resultados são dados em termos de 
Desempenho Mental e Motor. As escalas se destinam a crianças do nascimento até 42 meses de vida. A aplicação é realizada por meio de observação direta e interação com a criança de forma sistemática (CAMPOS; GONÇALVES; SANTOS, 2004; SILVA et al., 2011). Têm sido utilizadas por profissionais da saúde, sobretudo psicólogos (EICKMANN; LIRA; LIMA, 2002; FRAGA et al., 2008; GONTIJO et al., 2001; GOTO et al., 2005; LINHARES et al., 2000; MELLO; GONÇALVES; SOUZA, 2004).

As escalas Bayley têm sido utilizadas para avaliar o impacto de condições biológicas, como prematuridade e baixo peso, comparando com grupos controle, sem a condição (GOTO et al., 2005; MELLO; GONÇALVES; SOUZA, 2004), associando com variáveis ambientais (EICKMANN; LIRA; LIMA, 2002), ou com características maternas como ansiedade (FRAGA et al., 2008) e para avaliar procedimentos de intervenção junto a bebês muito prematuros (KOLDEWIJN et al., 2009).

Goto et al. (2005) utilizaram as Escalas Bayley-II para comparar o desenvolvimento de 67 crianças nascidas a termo, com peso adequado ou pequeno para a idade gestacional no $2^{\circ}$ mês de vida. Observaram diferenças significativas em todos os itens avaliados. Mello, Gonçalves e Souza (2004) utilizaram as Escalas Bayley-II para comparar o comportamento de lactentes nascidos a termo, com peso adequado, com lactantes pequenos para a idade gestacional no primeiro trimestre de vida. Os resultados identificaram diferenças significativas entre os grupos, principalmente nos itens: exploração de objetos e do ambiente; interação com o examinador e comportamentos motores específicos, como controle de movimentos e hipertonia muscular.

Eickmann, Lira e Lima (2002) utilizaram as Escalas Bayley-II para comparar o desenvolvimento motor e mental de crianças nascidas a termo, com baixo peso (menos de $2.500 \mathrm{~g}$ ) e com peso esperado (mais de $3.000 \mathrm{~g}$ ), correlacionando com variáveis demográficas e condições ambientais. Os resultados mostraram bom desempenho para as crianças com peso esperado, independentemente da condição socioeconômica, e para as com baixo peso de condição socioeconômica média. Observaram prejuízos para as crianças com baixo peso de condição socioeconômica baixa.

Fraga et al. (2008) utilizaram as Escalas Bayley-II para avaliar o desenvolvimento de 14 crianças nascidas pré-termo aos 12 meses, considerando a idade cronológica corrigida, verificando sua relação com a ansiedade materna, características neonatais e indicadores de desenvolvimento. Os itens relativos à resolução de problemas, permanência do objeto, intencionalidade, linguagem e motricidade correlacionaram-se significativamente com variáveis neonatais do bebê e com a ansiedade materna. A ansiedade materna mostrou ter efeito diferencial em áreas do desenvolvimento da criança, podendo atuar como fator 
de risco ao desenvolvimento motor amplo, mas como mecanismo de proteção ao desenvolvimento cognitivo.

Koldewijn et al. (2009) aplicaram as Escalas Bayley-II para avaliar um procedimento de intervenção oferecido a bebês muito prematuros, antes e depois do mesmo. A intervenção, que constava de orientações pontuais aos pais sobre desenvolvimento infantil, foi realizada em seis a oito visitas às casas de 86 bebês, até os seis meses de idade corrigida. Outras noventa crianças também foram avaliadas, mas, no mesmo espaço de tempo, receberam atendimento padrão. Os resultados obtidos apontaram para desempenho superior para os bebês submetidos à intervenção adicional em suas residências.

Pesquisadores utilizaram as Escalas Bayley de Desenvolvimento Infantil para verificar o impacto de condições sociais sobre o desenvolvimento de crianças saudáveis a partir do nascimento (ANDRACA et al., 1998; ANDRADE et al., 2005; SANTOS et al., 2008).

Andraca et al. (1998) utilizaram as Escalas Bayley-II para avaliar os efeitos de condições sociais e demográficas sobre o desenvolvimento de bebês até 12 meses de idade que nasceram em boas condições biológicas. Os resultados apontaram para decréscimo no desenvolvimento quanto piores as condições de risco. Andrade et al. (2005) utilizaram as Escala Bayley-II e o Inventário HOME para analisar a associação entre a qualidade do estímulo doméstico e o desempenho cognitivo infantil, identificando o impacto da escolaridade materna sobre a qualidade dessa estimulação. Participaram 350 crianças entre 17 e 42 meses. Os resultados mostraram que as crianças ocupando as primeiras ordens de nascimento, convivendo com reduzido número de menores de cinco anos, usufruem de melhor qualidade da estimulação no ambiente doméstico. Esse padrão de estimulação se mantém entre crianças que convivem com seus pais, cujas mães possuem melhor escolaridade, trabalham fora e convivem com companheiros no ambiente familiar. Santos et al. (2008) utilizaram as Escalas Bayley-II para avaliar o desenvolvimento de 320 crianças até 40 meses, associando com avaliações do ambiente realizadas por meio do Inventário HOME. Os resultados apontaram para o efeito indireto de fatores socioeconômicos, como falta de brinquedos e jogos apropriados para o desenvolvimento cognitivo da população estudada. Não foram observadas associações entre condição nutricional e desempenho cognitivo, um dos objetivos do estudo.

As Escalas Bayley-II foram usadas por Gontijo et al. (2001) para avaliar os efeitos de problemas de saúde sobre o desenvolvimento infantil. Os autores compararam o desempenho nas escalas de 28 crianças de zero a três anos, divididas em dois grupos, um deles formado por crianças com HIV+. Os resultados indicaram diferenças significativas nas habilidades motoras grossas e na linguagem. Os autores sugerem o acompanhamento sistemático de crianças infectadas. 
O Denver II Developmental Screening Test (traduzido como Teste de Triagem e Desenvolvimento Denver II, Teste de Denver II, Teste de Desenvolvimento de Denver II ou Escala Denver II) é um instrumento de triagem rápida, aplicado quando há suspeita de atraso no desenvolvimento ou a exposição a fatores de risco potenciais. Avalia as funções motora axial e apendicular, linguagem e aspectos pessoais e sociais, por meio de 125 itens aplicados, a partir de um kit padronizado, contendo um manual explicativo das provas. É prevista, também, a aplicação de um questionário prévio para os pais. Pontuam-se os itens em aprovado, falho, não aplicável e recusa. Após a soma dos aprovados, classificam-se em normal, suspeito e não testável. O teste abrange a faixa etária de um a 24 meses (CAMPOS; GONÇALVES; SANTOS, 2004). A proposta do instrumento é identificar crianças que necessitam de encaminhamento para serviços de estimulação precoce ou serviços especializados.

É um instrumento de triagem amplamente utilizado por profissionais da área da saúde, principalmente enfermeiros e pediatras, provavelmente por ser de fácil e rápida aplicação. Também por essa característica tem sido utilizado por pesquisadores, quando avaliam grandes populações, tendo em vista o efeito de variáveis socioambientais e biológicas ao nascer e ainda na primeira infância (HALPERN et al., 1996; HALPERN et al., 2000; HALPERN et al., 2008; POLLITT; KARIGER, 1996).

Halpern et al. (1996) utilizaram o Teste de Denver II para avaliar 20\% das crianças nascidas no ano de 1993, aos 12 meses de idade, numa cidade gaúcha de porte médio. Os resultados mostraram que 34\% das crianças avaliadas apresentaram desempenhos sugestivos para atraso no desenvolvimento, apontando uma associação entre a renda familiar baixa e baixo peso ao nascer. Sugerem a necessidade de triagem sistemática do desenvolvimento e programas de intervenção precoce em grupos de risco. Pollitt e Kariger (1996) acompanharam 1.363 recém-nascidos durante o primeiro ano de vida, sendo avaliada, entre outras variáveis, a relação entre desenvolvimento e aleitamento materno. Os resultados mostraram que, quanto maior o tempo de amamentação, menor o risco de atrasos no Teste de Denver II. Já as crianças que nunca foram amamentadas tiveram um risco $88 \%$ maior de apresentar atraso quando comparadas àquelas que mamaram por mais de seis meses. Com relação aos fatores de risco para atraso no desenvolvimento psicomotor, Halpern et al. (2000) pretenderam verificar a prevalência de atraso no desenvolvimento neuropsicomotor de crianças aos 12 meses de idade. Por meio da aplicação do Teste de Denver II, constataram que as crianças que tiveram dois ou mais itens de falha no teste e foram consideradas suspeitas de apresentarem atraso no desenvolvimento eram mais pobres, haviam nascido com peso menor, apresentavam idade gestacional menor do que 
37 semanas, tinham mais de três irmãos e haviam recebido leite materno por menos de três meses ou não haviam sido amamentadas.

Halpern et al. (2008) utilizaram o Teste de Denver II para comparar o desenvolvimento de dois grupos de crianças em condições semelhantes (oriundas de famílias de baixa renda) com intervalo de 10 anos. Um grupo foi avaliado em 1993 e outro, com as mesmas características, em 2003. Os autores observaram melhoras no desempenho geral avaliado para o último grupo, atribuindo o resultado observado às condições de saúde oferecidas, atualmente, à população de baixa renda.

O Teste de Denver II também foi utilizado para avaliar o desenvolvimento de crianças em um estudo longitudinal (REZENDE; BETELI; SANTOS, 2005) e para avaliar o efeito de condições biológicas adversas por ocasião do nascimento (CHERMONT et al., 2005; RESEGUE; PUCCINI; SILVA, 2008).

Rezende, Beteli e Santos (2005) utilizaram o Teste de Triagem de Desenvolvimento de Denver II para avaliar o desempenho de crianças de 0 a 4 anos em três creches paulistas, em dois anos consecutivos, num estudo longitudinal. Os resultados obtidos apontaram para prejuízos na área de linguagem para o grupo estudado. Chermont et al. (2005) utilizaram o Teste de Denver II para avaliar o desenvolvimento de 20 crianças nascidas prematuras e com peso inferior a $2.500 \mathrm{~g}$, nos primeiros dois anos de vida, atendidas no Programa de Seguimento de Recém-Nascidos Prematuros de um hospital universitário. Os resultados mostraram que $90 \%$ delas apresentaram atraso em pelo menos uma das quatro áreas avaliadas, com associações negativas com outras variáveis de risco, como idade das mães, pré-natal incompleto e fatores ambientais, como baixa renda. Resegue, Puccini e Silva (2008) utilizaram o Teste de Denver II para avaliar o desenvolvimento de 211 bebês que apresentavam um ou mais fatores de risco por ocasião do nascimento (baixo peso, prematuridade, filhos de mães adolescentes, com complicações neonatais etc.). Os resultados mostraram correlação negativa entre as variáveis estudadas (quanto menor o peso, maior o atraso observado).

Todavia, em alguns estudos a efetividade do instrumento foi contestada (FRAGA; LINHARES; CARVALHO, 2008; MAGALHÃES et al., 1999a; MAGALHÃES et al., 1999b), discutindo-se sua validade preditiva, uma vez que não é uma escala padronizada para a população brasileira. Magalhães et al. (1999a) utilizaram o Teste de Desenvolvimento de Denver (TDD) para avaliar crianças prematuras participantes de um programa de acompanhamento de desenvolvimento. Os resultados obtidos em alguns itens mostraram desempenho significativamente melhor do grupo de prematuros. Os autores indicam a pouca validade do instrumento para avaliar crianças, principalmente por compará-las com a amostra americana. Magalhães, Barbosa, Araújo, Paixão, Figueiredo e 
Gontijo (1999b) usaram o Teste de Desenvolvimento de Denver II para avaliar o desempenho de crianças nascidas prematuras, nas idades de 12, 18 e 24 meses. Os resultados obtidos mostraram que fatores culturais podem ter contribuído para o baixo desempenho dos sujeitos avaliados, recomendando cautela no uso deste instrumento com crianças brasileiras. Fraga, Linhares e Carvalho (2008) utilizaram o Teste de Denver II para avaliar os efeitos da ansiedade e da depressão materna no desenvolvimento de bebês prematuros no primeiro ano de vida. Os resultados não indicaram atrasos no desenvolvimento quando comparados os grupos de bebês com e sem mães depressivas. Os autores sugerem estudos com esse e com outros instrumentos de avaliação de desenvolvimento de bebês para validar ou não os resultados obtidos.

No entanto, pesquisadores têm associado a aplicação das Escalas Bayley e do Teste de Denver II, concomitantemente, para avaliar os efeitos da prematuridade (SCHIRMER; PORTUGUEZ; NUNES, 2006) e, sucessivamente, para a validação das Escalas Bayley para essa condição (ESPÍRITO SANTO; PORTUGUEZ; NUNES, 2009).

Schirmer, Portuguez e Nunes (2006) utilizaram o Teste de Denver II e as Escalas Bayley-II para avaliar o desenvolvimento cognitivo e de linguagem, aos três anos, de crianças que nasceram prematuras e eram acompanhadas no Ambulatório de Seguimento Neonatal. As crianças que nasceram menores apresentaram escores mais baixos nas Escalas Bayley-II e atrasos na área de linguagem avaliada pelo Teste de Denver II. Espírito Santo, Portuguez e Nunes (2009) compararam o desempenho no Teste de Denver II e na Escala Conners de 80 crianças nascidas prematuras e com baixo peso, aos quatro anos de idade. Os dados obtidos foram relacionados com peso ao nascer, idade gestacional, sexo e o desempenho nas Escalas Bayley-II, aplicada anteriormente. Os resultados apontaram diferenças estatisticamente significativas entre o baixo desempenho no Teste de Denver II e a presença de TDAH (Transtorno do Déficit de Atenção e Hiperatividade) e TDA (Transtorno de Déficit de Atenção), na Escala Conners. Comparando os resultados do Teste de Denver II com os já obtidos com a aplicação das Escalas Bayley-II, observaram resultados semelhantes, atestando a capacidade de predição das Escalas Bayley-II.

A escala desenvolvida por Pinto, Vilanova e Vieira (1997, 1998), denominada "Desenvolvimento do comportamento da criança no primeiro ano de vida", é, provavelmente, a escala mais recentemente padronizada no Brasil e tem se mostrado adequada para avaliar o desempenho de bebês. Este instrumento pretende avaliar o desenvolvimento de bebês por meio das habilidades sensoriais, motoras, de linguagem e de cognição a partir da sua interação com o meio ambiente. Avaliações mensais do desenvolvimento do bebê são conduzidas, comparando seu desempenho com o desempenho padrão estabe- 
lecido pela escala, possibilitando o planejamento de atividades que otimizem o seu desenvolvimento. Estas atividades, em geral, fazem parte de programas de estimulação precoce ou essencial que são desenvolvidos em parceria com outros profissionais e os adultos significativos ou cuidadores da criança, como pais, familiares ou educadores de creches. Preveem protocolos diferentes para meninas e meninos, para os 64 comportamentos avaliados, alocados de um a doze meses, a partir de critérios de estabilização, normalização e aparecimento.

Estudos têm sido conduzidos para avaliar condições de risco, como prematuridade e baixo peso, em crianças de até um ano de idade (GUIMAR ÃES et al., 2003; LINHARES et al., 2003; NOBRE et al., 2004) e para subsidiar a elaboração de programa de estimulação precoce (RODRIGUES, 2003b).

Linhares et al. (2003) avaliaram o desenvolvimento de crianças nascidas com baixo peso e muito baixo peso, aplicando, aos 11 meses, a "Escala do Desenvolvimento da criança no primeiro ano de vida" (EDCC) e encontraram diferenças significativas entre os dois grupos, sugerindo intervenção sistemática no decorrer do desenvolvimento, principalmente para o grupo de muito baixo peso. Nobre et al. (2004) avaliaram os efeitos da prematuridade no desenvolvimento de 38 crianças, aos seis meses de idade, utilizando a "Escala de Desenvolvimento da criança no primeiro ano de vida". O grupo 1 era de crianças prematuras, mas com enfermidades crônicas, e o 2 , sem prematuridade e enfermidade. Os resultados não apontaram diferenças entre os grupos relacionados à presença de problemas de saúde, mas em função da idade gestacional, sugerindo acompanhamento longitudinal. Guimarães et al. (2003) utilizaram a escala "O desenvolvimento da criança no primeiro ano de vida" para verificar o desenvolvimento neurossensório-motor aos quatro e aos seis meses de crianças prematuras. Os resultados apontaram para atrasos no desenvolvimento para todas as crianças avaliadas aos quatro meses e que, aos seis meses, ainda apresentavam defasagens comportamentais em sete dos comportamentos avaliados. Os autores sugerem que os resultados são indicadores de atraso no desenvolvimento para prematuros.

Rodrigues (2003a) acompanhou o desenvolvimento de 74 bebês de risco, 28 meninas e 46 meninos, durante o primeiro ano de vida, acrescido de orientações sistemáticas aos pais, mês a mês. Utilizou-se para as avaliações da escala "O desenvolvimento do comportamento da criança no primeiro ano de vida" (PINTO; VILANOVA; VIEIRA, 1997), que permitiu a observação de comportamentos que apareciam, normalizavam e estabilizavam para cada uma das idades/mês. Para as orientações aos pais foi utilizado o programa "Brinquedos e brincadeiras para o bebê" (PEREZ-RAMOS; PÊRA; MAIA, 1995). Os resultados mostraram que a maioria dos bebês apresentou desenvolvimento dentro do esperado para sua idade cronológica, sendo que as meninas apresen- 
taram desempenho em desenvolvimento psicológico pouco acima dos meninos na amostra estudada.

Outra escala que vem sendo utilizada é a EADP - Escala de Avaliação do Desenvolvimento Psicomotor de 0 a 24 meses (RODRIGUEZ et al., 1992), que avalia o rendimento da criança frente a situações que, para serem resolvidas, requerem determinado grau de desenvolvimento psicomotor. A escala avalia quatro áreas de funcionamento: motora, linguagem, social e coordenação óculo-manual, sendo a linguagem dividida em compreensiva e expressiva (MELCHIORI et al., 2003), em 75 itens.

Pesquisadores compararam a efetividade desta escala com as Escalas Bayley (SILVA; CURSINO; DIAS, 1993). Os autores realizaram um estudo comparando duas escalas reduzidas: a Escala de Desenvolvimento de Heloisa Marinho e a EADP (Escala de Avaliação do Desenvolvimento Psicomotor) com um instrumento que é reconhecido como eficiente, as Escalas Bayley de Desenvolvimento Infantil, para avaliar a efetividade de uma das escalas na identificação de atrasos no desenvolvimento infantil, mas que fosse de fácil aplicação em Unidades Básicas de Saúde. Os resultados da EADP se assemelharam aos da Bayley, indicando sua utilização em contextos em que é necessário avaliar muitas crianças em um curto espaço de tempo, otimizando as orientações aos pais e/ou cuidadores.

A Alberta Infant Motor Scale (AIMS) foi construída por Piper e Darrah, em 1994 (citado por CAMPOS; GONÇALVEZ; SANTOS, 2004), e tem como objetivo avaliar a maturação motora de crianças até os 18 meses, quando há a locomoção independente, por meio de 58 itens. Ao final da avaliação, o desempenho da criança é comparado com dados normativos para as diferentes idades. Tem sido utilizada para comparar o desenvolvimento motor de crianças prematuras com crianças a termo (CAMPOS et al., 2007; CASTRO et al., 2007; MANACERO; NUNES, 2008). Por se tratar de uma escala relativamente nova, pesquisas têm sido conduzidas para verificar sua validade para a população brasileira (ALMEIDA et al., 2008; CAMPOS et al., 2006).

Campos et al. (2007) utilizaram a Alberta Infant Motor Scale (AIMS) para realizar um estudo longitudinal, comparando o controle postural de lactentes nascidos a termo, pequenos (PIG) e adequados para a idade gestacional (AIG) aos três, seis, nove e 12 meses. Os resultados obtidos permitiram concluir que o controle postural foi similar nos grupos e atribuem os resultados aos possíveis efeitos do trabalho materno e da creche, locais de frequência diária da maioria dos bebês PIG. Manacero e Nunes (2008) também não observaram diferenças entre prematuros e a termo em desenvolvimento motor, avaliados aos quatro e aos oito meses de idade corrigida, utilizando a AIMS. 
Resultados diferentes foram obtidos por Castro, Lima, Aquino e Eickmann (2007), que utilizaram a AIMS (Alberta Infant Motor Scale) para avaliar o desenvolvimento motor de crianças nascidas prematuras e encontraram diferenças significativas entre bebês nascidos entre 29 e 34 semanas e bebês nascidos entre 35 e 36 semanas, apontando a idade gestacional dos bebês como uma variável importante para risco ao desenvolvimento motor. Campos et al. (2006) avaliaram 43 bebês nascidos a termo, sem fatores de risco identificados, para verificar a validade da Alberta Infant Motor Scale (AIMS), comparando-a com a Bayley Scales of Infant Development II (BSID-II), no sexto mês de idade. Os resultados sugerem boa concordância entre os instrumentos de avaliação utilizados. Almeida, Dutra, Mello, Reis e Martins (2008) também conduziram um estudo avaliando 88 crianças prematuras aos seis e aos 12 meses, com o objetivo de verificar a validade interobservadores para os itens da escala, comparando-a com os itens das Escalas Bayley-III. Concluíram que a AIMS é uma escala confiável para ser utilizada na avaliação de bebês de risco, com a vantagem de ser de fácil aplicação.

O instrumento Movement Assessment of Infant (MAI) foi elaborado com o objetivo de identificar bebês com risco para anormalidades neurológicas e, entre elas, a paralisia cerebral. Posteriormente, mostrou-se útil para subsidiar programas de estimulação precoce. Sua aplicação é baseada em critérios obtidos a partir da manipulação da criança e observação direta da mesma. É prevista a aplicação em crianças até 12 meses (CAMPOS; GONÇALVES; SANTOS, 2004).

Esse instrumento tem sido objeto de validação de pesquisadores na área de fisioterapia com ênfase para a detecção precoce de problemas neurológicos em bebês prematuros (CARDOSO et al., 2004; MAGALHÃES et al., 2001).

Magalhães et al. (2001) avaliaram a validade da Escala MAI (Movement Assessment of the Infant) para identificar paralisia cerebral em bebês pré-termo. Entre as variáveis observadas, as significativas para paralisia cerebral foram: idade gestacional, sexo masculino e presença de intercorrências neurológicas. Os autores indicam a escala para essa finalidade. Cardoso et al. (2004) realizaram um estudo para validar a Movement Assessment of the Infants (MAI) para detecção precoce de paralisia cerebral em crianças prematuras (menos de 32 semanas) e baixo peso (inferior a $1.500 \mathrm{~g}$ ). A escala mostrou-se efetiva, se aplicada por volta de oito meses de idade, corrigida por escores iguais ou maiores que 13 pontos. É sensível, também, para prever problemas de coordenação motora na idade escolar para crianças com 10 pontos ou mais.

Outro instrumento identificado para avaliar desempenho de prematuros foi o Protocolo de Observação do Desenvolvimento Cognitivo e de Linguagem Expressiva. Bühler, Flabiano, Mendes e Limongi (2007) utilizaram o referido protocolo, elaborado com base nos estudos de Piaget, para avaliar o 
desenvolvimento cognitivo de 22 crianças, das quais oito nascidas pré-termo, dos seis aos 12 meses de idade. Os resultados indicaram diferenças estatisticamente significantes entre os dois grupos para todas as fases da construção da permanência do objeto, evidenciando o atraso (média de 2 a 3 meses de atraso) no desenvolvimento cognitivo, reforçando a ideia de que as crianças nascidas pré-termo e de muito baixo peso apresentam risco para alterações ou atrasos, justificando a elaboração de programas de intervenção junto a essas crianças já durante o primeiro ano de vida.

Os instrumentos até então descritos apresentam limitações, dependendo do uso dos mesmos. Para avaliações do desenvolvimento que pretendem subsidiar a eleição de bebês expostos a condições de risco em programas de estimulação precoce, aqueles que se propõem a avaliações rápidas, como o Teste de Denver, a EADP e a Escala do desenvolvimento do bebê no primeiro ano de vida, não seriam os indicados por possuírem poucos comportamentos. Outra questão a ser considerada é a especificidade de instrumentos como a Alberta Infant Motor Scale e a Movement Assessment of the Infants que, apesar de avaliar consistentemente o repertório motor do bebê, não contemplam as demais áreas, impossibilitando a organização de um programa mais abrangente.

A extensão do instrumento é uma variável a ser considerada no trabalho com crianças de risco. Alguns autores têm sugerido o acompanhamento de crianças até a idade escolar, principalmente aquelas que nascem prematuras (CHERMONT et al., 2005; HADDERS-ALGRA, 2004; MAGALHÃES et al., 1999a; 1999b; MÉIO; LOPES; MORSH, 2003). Portanto, a utilização de instrumentos que avaliam a criança até um, dois ou três anos inviabiliza o acompanhamento do seu desenvolvimento posterior.

O IPO - Inventário Portage Operacionalizado (WILLIAMS; AIELLO, 2001), recentemente adaptado para a realidade brasileira, é composto por 580 comportamentos distribuídos em cinco áreas, separados por faixa etária de zero a seis anos e uma área específica para bebês de zero a quatro meses. As áreas de Cognição (avalia a linguagem receptiva e o estabelecimento de relações de semelhanças e diferenças), Socialização (avalia habilidades relevantes na interação com as pessoas), Autocuidado (avalia a independência para o alimentar-se, vestir-se, banhar-se etc.), Linguagem (avalia o comportamento verbal expressivo) e Desenvolvimento Motor (avalia os movimentos coordenados por pequenos e grandes músculos) compõem o conjunto de áreas e comportamentos avaliados pelo IPO. Uma vantagem adicional deste instrumento é a possibilidade de avaliar as crianças até seis anos de idade.

Para a avaliação de bebês de risco, o IPO tem-se mostrado eficiente. Estudos têm sido conduzidos para avaliar o desenvolvimento de bebês prematuros (LAMÔNICA; PICOLONI, 2008), com outras condições biológicas de 
risco (YOSHIDA; RODRIGUES, 2008) e, ainda, um estudo com o objetivo de identificar comportamentos típicos de bebês, a cada mês (TAQUES; RODRIGUES, 2006). Lamônica e Picolini (2008) utilizaram o Inventário Portage Operacionalizado para verificar o desempenho de habilidades do desenvolvimento linguístico, cognitivo, motor, de autocuidados e socialização em 30 crianças prematuras, de ambos os sexos, de 6 a 24 meses. Os resultados indicaram que as áreas mais defasadas do grupo, na faixa etária de 6 a 12 meses, foram linguagem e autocuidados e, na faixa etária de 12 a 24 meses, foram linguagem, cognição e autocuidados. Os autores sugerem o acompanhamento rigoroso de recém-nascidos prematuros, por meio do desenvolvimento de programas de acompanhamento com equipe multidisciplinar.

Taques e Rodrigues (2006) aplicaram o Inventário Portage Operacionalizado (IPO) em 40 bebês, com o objetivo de verificar se havia diferenças na ocorrência a cada mês, durante quatro meses, dos 45 comportamentos previstos no Protocolo de Estimulação Infantil. Esses autores concluíram que há diferenças entre os gêneros e que alguns comportamentos ocorrem tipicamente a cada mês, sugerindo adequações na escala. Concluem que um número menor de comportamentos avaliados a cada mês facilitaria o processo de avaliação de bebês e orientação de pais. Contudo, ressaltam que o Inventário Portage Operacionalizado tem se mostrado um instrumento importante de avaliação de desenvolvimento de bebês, considerando o grande número de comportamentos avaliados, não presentes em outras escalas.

Yoshida e Rodrigues (2008) desenvolveram um estudo com o objetivo de avaliar a influência de condições biológicas de risco (prematuridade, baixo peso, malformações) no desenvolvimento de bebês utilizando o IPO. Os resultados analisados indicaram que, independente do sexo, as médias de desempenho mais baixas foram as dos bebês prematuros.

A avaliação de bebês de até um ano, pelo Inventário Portage Operacionalizado, compreende um rol de 110 comportamentos e mais 45 comportamentos do Protocolo de Estimulação Infantil. Porém, não prevê quantos e quais comportamentos deveriam ocorrer a cada mês, o que permitiria a identificação de defasagens de repertórios comportamentais precocemente, facilitando a orientação aos pais e a elaboração de programas de estimulação precoce.

Estudos foram implementados para analisar o desenvolvimento de comportamentos típicos, a partir da alocação dos 45 comportamentos do Protocolo de Estimulação Infantil, nas áreas previstas do IPO original, como forma de acompanhar o desenvolvimento de bebês e facilitando a orientação aos pais. Embora esse movimento ainda não tenha sido feito de forma sistemática, alguns resultados têm apontado para a pertinência dessa medida (RODRIGUES, 2009; TIRELLI, 2008; YAMADA, 2007; YOSHIDA, 2008). 
Rodrigues (2009) realizou um estudo, utilizando o Inventário Portage Operacionalizado, que apontou para diferenças entre o desempenho de bebês comparando bebês prematuros, baixo peso, filhos de mães adolescentes e com o grupo controle. Os dados indicaram prejuízo para os bebês prematuros e com baixo peso, nascidos a termo, quando comparados com bebês filhos de mães adolescentes e do grupo controle.

Yamada (2007) desenvolveu um estudo com 40 bebês, sendo 20 de cada sexo, com o objetivo de identificar comportamentos típicos a cada mês, considerando o sexo do bebê, utilizando o Inventário Portage Operacionalizado adaptado. Nos resultados obtidos, observa-se que alguns comportamentos, a cada mês, deverão ser avaliados, tanto para meninos como para meninas. Outros o serão somente para um dos gêneros. Todavia, há diferenças quanto aos comportamentos que aparecem, normalizam e estabilizam. Os comportamentos passíveis de intervenção junto aos pais, organizando o ambiente para que eles se manifestem, são os comportamentos que, a cada mês, normalizam ou estabilizam. Tirelli (2008) acompanhou o desenvolvimento de 60 bebês até três meses de idade, também com o objetivo de identificar diferenças entre o desempenho de meninos e meninas, a cada mês, avaliados com o Inventário Portage Operacionalizado adaptado. Todavia, apesar de identificar diferenças entre os sexos, a autora julgou que os participantes não foram em número suficiente para justificar a elaboração de protocolos separados. Yoshida (2008) avaliou 96 bebês, 46 meninos e 50 meninas, de um a seis meses de idade, utilizando o Inventário Portage Operacionalizado adaptado, com os seguintes objetivos: identificar comportamentos típicos para bebês nessa faixa etária; averiguar diferenças comportamentais entre os sexos considerando a condição de risco que apresentam (prematuridade, baixo peso, bebês filhos de mães com HIV+, filhos de mães adolescentes). Os resultados mostraram que é possível identificar comportamentos típicos a cada mês e que há diferença entre os sexos em Cognição, Socialização e Desenvolvimento Motor e nenhuma diferença em Linguagem e Autocuidado. Com relação à condição de risco, observou-se que, à medida que o bebê cresce, aumentam as diferenças dos bebês com alguma condição de risco, quando comparados com o Grupo Controle. Observou-se, no entanto, que o desempenho de bebês filhos de mães adolescentes é semelhante ao dos bebês do grupo controle.

Os resultados obtidos com o Inventário Portage Operacionalizado têm mostrado que ele é um instrumento que pode ser utilizado para a identificação de defasagens que servirão como ponto de partida para a orientação de pais ou para a elaboração de programas de intervenção junto a bebês. Justifica-se sua utilização na identificação de prejuízos no desenvolvimento de bebês de risco, o que pode nortear a elaboração de programas específicos para atender às demandas identificadas, seja em termos do caráter do programa, ou de sua 
extensão. Outro ponto importante é adequar o Inventário Portage Operacionalizado para um trabalho efetivo com a população de bebês, no primeiro ano de vida, a partir da identificação de comportamentos típicos a cada mês ou, pelo menos, em períodos mais curtos do que o apresentado pelo instrumento que vai de zero a um ano de idade.

Nunes, Sisdelli e Fernandes (1994) chamam a atenção para o estudo dos instrumentos que avaliam o desenvolvimento de bebês, pela sua validade preditiva e pela possibilidade de organização de programas de estimulação precoce. Porém, é preciso adequá-los e, se possível, validá-los, ou, ainda, padronizá-los para a população brasileira.

\section{REFERÊNCIAS}

ALMEIDA, K. M. A.; DUTRA, M. V. P.; MELLO, R. R.; REIS, A. B. R.; MARTINS, P. S. M. Validade concorrente e confiabilidade da Alberta Infant Motor Scale em lactentes nascidos prematuros. Jornal de Pediatria, v. 84, n. 5, p. 442-448, 2008.

ALVES, P. P.; FERREIRA, M. F. R.; NUNES, L. R. O. P.; OLIVEIRA, M. C. B.; KAORU, J.; EPELBOIM, S. O desenvolvimento cognitivo de bebês prematuros e alguns aspectos neuromotores associados. Pediatria Moderna, v. XXXIII, n. 7, p. 511-530, 1997.

ANDRACA, F.; PINO, P.; LA PARRA, A.; RIVERA, F.; CASTILLO, M. Fatores de riesgo para el desarrollo psicomotor em lactantes nacidos em optimas condiciones biológicas. Revista de Saúde Pública, v. 32, n. 2, p. 138-147, 1998.

ANDRADE, S. A.; SANTOS, D. N.; BASTOS, A. C.; PEDROMÔNICO, M. R. M.; ALMEIDA-FILHO, N.; BARRETO, M. L. Ambiente familiar e desenvolvimento cognitivo infantil: uma abordagem epidemiológica. Revista de Saúde Pública, v. 39, n. 4, p. 606-611, 2005.

BÜHLER, K. E. B.; FLABIANO, F. C.; MENDES, A. E.; LIMONGI, S. C. O. Construção da permanência do objeto em crianças nascidas pré-termo muito baixo peso. Revista CEFAC, v. 9, n. 3, p. 300-307, 2007.

CAMPOS, D.; SANTOS, D. C. C.; GONÇALVES, V. M. G.; GOTO, M. M. F.; ARIAS, A. V.; BRIANEZE, A. C. G. S.; MELLO, T. M. Concordância entre escalas de triagem e diagnóstico do desenvolvimento motor no sexto mês de vida. Jornal de Pediatria, v. 82, n. 6, p. 470-474, 2006.

; MONTEBELO, M. I. L.; GOTO, M. M. F.; GABBARD,

C. Controle postural de lactentes nascidos a termo pequenos para a idade gestacional, Revista Brasileira de Fisioterapia, v. 11, n. 1, p. 7-12, 2007. 
CAMPOS, T. M.; GONÇALVES, V. M. G.; SANTOS, D. C. C. Escalas padronizadas de avaliação do desenvolvimento motor de lactentes. Temas sobre Desenvolvimento, v. 13, n. 77, p. 5-11, 2004.

CARDOSO, A. A.; MAGALHÃES, L. C.; AMORIM, R. H. C.; PAIXÃO, M. L.; MANCINI, M. C.; ROSSI, L. D. F. Validade preditiva do Movement Assessment of Infants para crianças pré-termo brasileiras. Arquivos de Neuropsiquiatria, v. 62, n. 4, p. 52-57, 2004.

CASTRO, A. G.; LIMA, M. C.; AQUINO, R. R.; EICKMANN, S. H. Desenvolvimento do sistema sensório motor oral e motor global em lactentes pré-termo. Pró-Fono Revista de Atualização Científica, v. 19, n. 1, 29-38, 2007.

CHERMONT, A. G.; CUNHA, M. S.; SALES, L. M. M.; MORAES, A. N.; MALVEIRA, S. S. Avaliação do desenvolvimento, pela escala de Denver II, de recém-nascidos prematuros de baixo peso. Revista CEFAC, p. 19, n. 2, p. 59-66, 2005.

CHIARATTI, P. S.; SPROCATTI, R.; PIOVESANA, A. M. S. G. Crianças de alto risco: evolução de aspectos linguísticos e cognitivos. Temas sobre Desenvolvimento, v. 10, n. 57, p. 19-23, 2001.

EICKMANN, S. H.; LIRA, P. I. C.; LIMA, M. C. Desenvolvimento mental e motor aos 24 meses de crianças nascidas a termo com baixo peso. Arquivos de Neuropsiquiatria, v. 60, n. 3-B, p. 748-754, 2002.

ESPÍRITO SANTO, J. L.; PORTUGUEZ, M. W.; NUNES, M. L. Status cognitivo-comportamental de prematuros baixo peso ao nascimento em idade pré-escolar que vivem em país em desenvolvimento. Jornal de Pediatria, v. 85, n. 1, p. 35-41, 2009.

FRAGA, D. A.; LINHARES, M. B. M.; CARVALHO, A. E. V.; MARTINEZ, F. E. Desenvolvimento de bebês prematuros relacionado a variáveis neonatais e maternas. Psicologia em Estudo, v. 13, n. 2, p. 335-344, 2008.

GONTIJO, A. P. B.; SCOD, M. C. M.; FÓSCOLO, D. R. C.; MOREIRA, T. G. AIDS pediátrica: características do neurodesenvolvimento. Revista Paulista de Pediatria, v. 19 , n. 3, p. 127-132, 2001.

GOTO, M. M. F.; GONÇALVES, V. M. G.; ARANHA NETTO, A.; MORCILlO, A. M.; MOURA-RIBEIRO, M. V. L. Neurodesenvolvimento de lactentes nascidos a termo pequenos para a idade gestacional no segundo mês de vida. Arquivos de Neuropsiquiatria, v. 63 , n. 1, p. $75-82,2005$.

GUimarÃES, E. L.; SANTOS, A. P.; CASTRO, A. M.; GOMES, K. M.; FARIAS, L. C. A.; OLIVEIRA, M. C.; TUDELLA, E. Estudo comparativo do desenvolvimento neurossensório motor de recém-nato pré-termo aos quatro e seis meses de vida, segundo a escala "O desenvolvimento do comportamento da criança no primeiro ano de vida". Fisioterapia e Movimento, v. 16, n. 4, p. 41-46, 2003.

HADDERS-ALGRA, M. General movements: A window for early identification of children at high risk for developmental disorders. Jornal de Pediatria, v. 145, n. 2, p. 12-18, 2004. 
HALPERN, R.; BARROS, A. J. D.; MATIJASEVICH, A.; SANTOS, I. S.; VICTORA, C. G.; BARROS, F. C. Development status at age 12 months according to bird weight and family income: a comparison of two Brazilian birth cohorts. Cadernos de Saúde Pública, v. 24, n. 3, p. 444-450, 2008.

; BARROS, F. C.; HORTA, B. L.; VICTORA, C. G. Desenvolvimento neuropsicomotor aos 12 meses de idade em uma coorte de base populacional no Sul do Brasil: diferenciais conforme peso ao nascer e renda familiar. Cadernos de Saúde Pública, v. 12, n. 1, 73-78, 1996.

; GIUGLIANI, E. R. J.; VICTORA, C. G.; BARROS, F. C.; HORTA, B. L. Fatores de risco para suspeita de atraso no desenvolvimento neuropsicomotor aos 12 meses de vida. Jornal de Pediatria, v. 76, n. 6, p. 421-428, 2000.

KOLDEWIJN, K.; WOLF, M. J.; VAN WASSENAER, A.; MEIJSSEN, D.; VAN SONDEREN, L.; VAN BAAR, A.; BEELEN,A.; NOLLET, F.; KOK, J.J. The infant behavioral assessment and intervention program for very low birth weight infants at 6 months corrected age. Pediatric, v. 154, n. 1, p. 33-38, 2009.

LAMÔNICA, D. A. C.; PICOLINI, M. M. Habilidades do desenvolvimento de prematuros. Revista CEFAC, 2008. Disponível em: <http://www.scielo.br/pdf/rcefac/v11s2/51-08. pdf $>$. Acesso em: 26/02/2011.

LINHARES, M. B. M.; CARVALHO, A. E. V.; BORDIN, M. B. M.; JORGE, S. M. Suporte psicológico ao desenvolvimento de bebês pré-termo com peso de nascimento $<1500 \mathrm{~g}$, na UTI e no seguimento longitudinal. Temas em Psicologia, v. 7, n. 3, p. 245-262, 1999.

; CHIMELlO, J. T.; MARTINEZ, F. E.; JORGE, S. M. Prematuridade e muito baixo peso como fatores de risco ao desenvolvimento da criança. Cadernos de Psicologia e Educação - Paidéia, v. 10, n. 12, p. 60-69. 2000.

; MACHADO, C.; MARTINEZ, F. E. Desenvolvimento de bebês nascidos pré-termo no primeiro ano de vida. Cadernos de Psicologia e Educação - Paidéia, v. 13, n. 25, p. 59-72, 2003.

MAGAlhãeS, L. C.; BARBOSA, V. M.; LOPES, K. C.; PAIXÃO, M. L. Estudo longitudinal do desenvolvimento de recém-nascidos pré-termo: avaliação na idade pré-escolar. Revista Brasileira de Neurologia, v. 35, p. 87-93, 1999a.

; ___ ; ARAÚJO, A. R.; PAIXÃO, M. L., FIGUEIREDO, E. M.; GONTIJO,

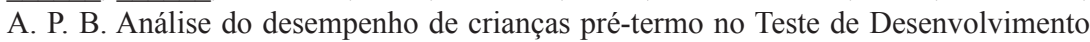
de Denver nas idades de 12, 18 e 24 meses. Pediatria, v. 21, n. 4, p. 330-339, 1999b.

; AMORIN, F. P.; PAIXÃO, M. L.; BARBOSA, V. M.; MANCINI, M. C. Influência de fatores de risco biológico nos escores de um teste para detecção de paralisia cerebral em crianças pré-termo. Temas sobre Desenvolvimento, v. 10, n. 58-9, p. 5-12, 2001.

MANACERO, S.; NUNES, M. L. Avaliação do desempenho motor de prematuros nos primeiros meses de vida na Escala Motora Infantil de Alberta (AIMS). Jornal de Pediatria, v. 84, n. 1, p. 53-59, 2008. 
MARTINS, I. M. B.; LINHARES, M. B.; MARTINEZ, F. E. Indicadores de desenvolvimento na fase pré-escolar de crianças nascidas pré-termo. Psicologia em Estudo, v. 10, n. 2, p. 20-32, 2005.

MAZETTO, M. D. C.; RODRIGUES, O. M. P. R.; MELCHIORI, L. E.; YAMADA, M. O. (submetido) Adequação de uma escala de desenvolvimento para uso em triagem diagnóstica para implante coclear. Revista Brasileira de Crescimento e Desenvolvimento Humano.

MÉIO, M. D. B. B.; LOPES, C. S.; MORSCH, D. S. Fatores prognósticos para o desenvolvimento cognitivo de prematuros de muito baixo peso. Revista de Saúde Pública, v. 37, n. 3, p. 311-318, 2003.

MELCHIORI, L. E. et al. A utilização de instrumentos de avaliação do desenvolvimento infantil em creches municipais. In: REUNIÃO ANUAL DA SOCIEDADE BRASILEIRA DE PSICOLOGIA, 33., 2003, Belo Horizonte. Anais. SBP, p. 162-163, 2003.

MELlO, B. B. A.; GONÇALVES, V. M. G.; SOUZA, E. A. P. Comportamento de lactentes nascidos a termo pequenos para a idade gestacional no primeiro trimestre da vida. Arquivos de Neuropsiquiatria, v. 62, n. 4, 2004.

NOBRE, F. D. A.; LINHARES, M. B. M.; CARVALHO, A. E. V.; PANAIA, C. C.; FERLIN, M. L.; MARTINEZ, F. E. Indicadores de desenvolvimento inicial e saúde em crianças pré-termo de muito baixo peso com enfermidade crônica. Resumo. In: REUNIÃO ANUAL DA SOCIEDADE BRASILEIRA DE PSICOLOGIA, 34, 2004. Anais... p. 46, 2004.

NUNES, L. R. D. P.; SISDELLI, R. O.; FERNANDES, R. L. C. O valor dos testes de bebês e suas implicações para a Psicologia do Desenvolvimento. Revista Brasileira de Educação Especial, v. 5, p. 107-125, 1995.

PEREZ-RAMOS, A. M. Q.; PÊRA, C.; MAIA, M. A. Brinquedos e brincadeiras para o bebê nos seus dois primeiros anos de vida: manual de orientação. São Paulo: Vetor, 1995.

PINTO, E. B.; VILANOVA, L. C. P.; VIEIRA; R. M. O desenvolvimento do comportamento da criança no primeiro ano de vida: padronização de uma escala para avaliação e acompanhamento. São Paulo: Casa do Psicólogo/FAPESP, 1997.

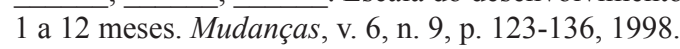

POLLITT E.; KARIGER, P. Breastfeeding and child development. Food Nutrition Bulletin, v. 17, n. 4, p. 401-418, 1996.

RESEGUE, R.; PUCCINI, R. F.; SILVA, E. M. K. Risk factors associated with developmental abnormalities among high-risk children attended at a multidisciplinary clinic. Medicine Journal, v. 126, n. 1, p. 4-10, 2008.

REZENDE, M. A.; BETELI, V. C.; SANTOS, J. L. F. Avaliação de habilidades de linguagem e pessoal-sociais pelo teste Denver II em instituições de Educação Infantil. Acta Paulista de Enfermagem, v. 18, n. 1, p. 56-63, 2005. 
RODRIGUES, O. M. P. R. Acompanhamento do desenvolvimento de bebês de risco: avaliação e orientação aos pais. Relatório trienal. Departamento de Psicologia, Faculdade de Ciências, UNESP, Bauru, 2003a.

. Bebês de risco e sua família: o trabalho preventivo. Temas em Psicologia, v. 2, p. 34-45, 2003b.

. O Inventário Portage Operacionalizado e o desenvolvimento de bebês. Tese (Livre-Docência) - Universidade Estadual Paulista Julio de Mesquita Filho (UNESP). Bauru/SP, 2009.

RODRIGUEZ, S.; ARANCIBIA, V.; UNDURRAGA, C. Escala de evaluación del desarrollo psicomotor de 0 a 24 meses. Santiago (Chile): Galdoc, 1992.

SANTOS, L. M.; SANTOS, D. N.; BASTOS, A. C. S.; ASSIS, A. M. O.; PRADO, M. S.; BARRETO, M. L. Determinants of early cognitive development: hierarchical analysis of a longitudinal study. Cadernos de Saúde Pública, v. 24, n. 2, p. 427-437, 2008.

SCHIRMER, C. R.; PORTUGUEZ, M. W.; NUNES, M. L. Avaliação da evolução dos aspectos linguísticos em crianças que nasceram prematuras aos três anos de idade. $A r$ quivos de Neuropsiquiatria, v. 64, n. 4, p. 926-931, 2006.

SILVA, N. D. S. H. et al . Instrumentos de avaliação do desenvolvimento infantil de recém-nascidos prematuros. Revista Brasileira de Crescimento e Desenvolvimento Humano, São Paulo, v. 21, n. 1, p. 85-98, 2011.

SILVA, R. C.; CURSINO, E. A.; DIAS, L. B. S. Acompanhamento do desenvolvimento psicomotor infantil em unidades básicas de saúde. Psicologia: Teoria e Pesquisa, v. 9, n. 3, p. 521-534, 1993.

TAQUES, D. C. S. R.; RODRIGUES, O. M. P. R. Avaliação do repertório comportamental de bebês nos quatro primeiros meses de vida: uma proposta de análise. Revista Brasileira de Crescimento e Desenvolvimento Humano, v. 16, n. 2, p. 77-87, 2006.

TIRELLI, R. A. Avaliação do repertório comportamental de bebês e o Inventário Portage Operacionalizado. Relatório final de Iniciação Científica. CNPq. (PIBIC), 2008.

WILliamS, L. C. A.; AIEllo, A. L. R. O Inventário Portage Operacionalizado: intervenção com famílias. São Paulo: Memnon/FAPESP, 2001.

YAMADA, J. K. Inventário Portage Operacionalizado: avaliação do repertório comportamental de bebês. Relatório final de Iniciação Científica. FAPESP/06/58853-5, 2007.

YOSHIDA, M. L. A. Inventário Portage Operacionalizado: avaliação do repertório comportamental de bebês de zero a seis meses de idade. Relatório final de Iniciação Científica. FAPESP, n. 59973-7, 2008.

; RODRIGUES, O. M. P. R. Condições de risco determinando o desenvolvimento de bebês. In: JORNADA DE ANÁLISE DO COMPORTAMENTO DA UFSCar, 7. 
RODRIGUES, O. M. P. R. Escalas de desenvolvimento infantil e o uso com bebês

São Carlos, 2008. Anais... Disponível em: < http://www.redepsi.com.br/portal/modules/ smartsection/item.php?itemid=1411>. Acesso em: 20/10/2011.

Texto recebido em 25 de agosto de 2011.

Texto aprovado em 28 de outubro de 2011. 